\title{
Measurement and Visualisation of Micron - Scale Strain Distributions in Aluminum Alloys
}

\author{
Don Steele and D.J. Lloyd
}

Alcan International R\&D, Box 8400, Kingston, Ontario Canada, K7L 5L9

The forming of $\mathrm{Al}$ alloys in applications such as automotive involve avariety of steps and strain paths. In order to fully understand and model the formability of materials, a measurement of the development of strain during the forming process is important, particularly in regards to the distribution of strain and its localisation. Since the development of strain is influenced by the microstructure, and microstructural features such as grain size and grain orientation, the measurement of strain has to be at the subgrain level. Bending is a particularly important deformation mode in many forming operations, and the literature contains several references to the development of strain during bending [1-3]. A technique has been developed to measure and visualize the local strain at the subgrain level after bending, which can then be compared with plasticity models.

The difficulty in measuring strain at this level is in the creation of an ultrafine fiduciary system which will withstand the deformation process. The SEM provides a potential marking mechanism in the form of precisely deposited carbon contamination spots. A regular grid of contamination spots is easily created in an automated fashion with the use of EDXS beam control software, and these spots are found to survive to high strain levels. Figure 1 shows such a spot pattern on a sheet surface before and after bending. The cells defined by the fiducials can be measured before and after bending to determine the true strain.

Once the coordinates of the fiducials defining the cells are registered using image analysi software, measurement is rapid using an automation macro. A pseudocolour lookup table where colours correspond to different strain levels is applied as an overlay to aid in the visualisation of the strain distribution. Figure 2 shows the distribution of strain through the cross section of a wrap bend test specimen. The technique has been used at Alcan International to verify finite element models (FEM) describing the maximum strains obtained at the surface of 6XXX aluminum automotive sheet with various degrees of bending. Figure 3 shows the agreement of empirical measurements with one such FEM.

Despite the close agreement with the FEM, observation of the bend surface shows out of plane displacement. This will affect the measurement due to parallax, and future work will include topographic measurements of the strain measurement region. The influence and development of crystallographic texture on measured local strain is also being investigated via EBSD, which can be carried out concurrently with the grid deposition.

\section{References}

[1] N. Triantafyllids and A. Needleman, Int. J. Solids Structures, 1982, vol.18, pp. 121-138.

[2] L.R. Morris et al., Microstructural Science, 1979, vol. 7, pp. $59-67$.

[3] G.J. Geist and B.A. Parker, J. Mech. Working Techn., 1984, vol. 9, pp. 201-207. 

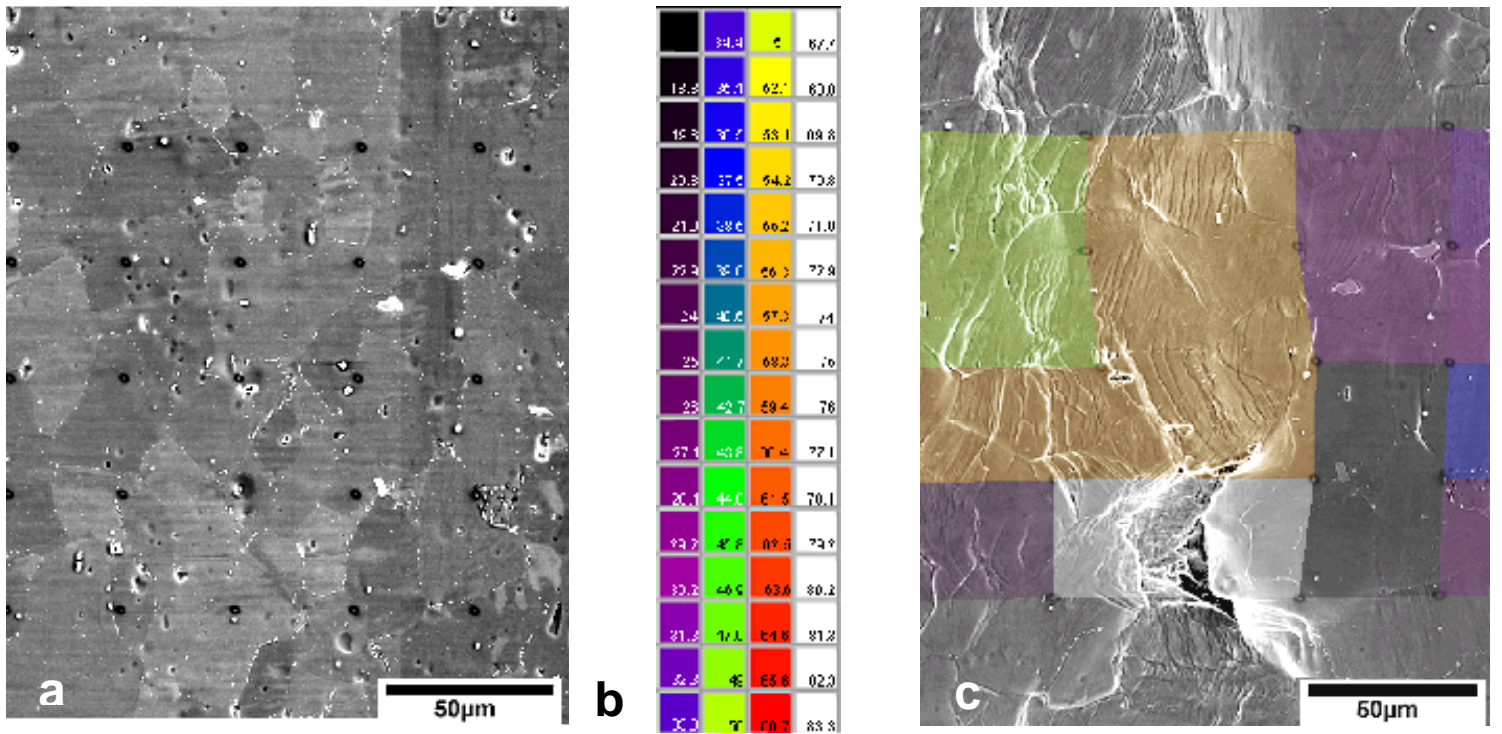

FIG. 1. SEM deposited contamination grid at the surface of a bend test specimen before a) and the same area after c) bending, and the corresponding true strain indexed colour gradient $b$ ).

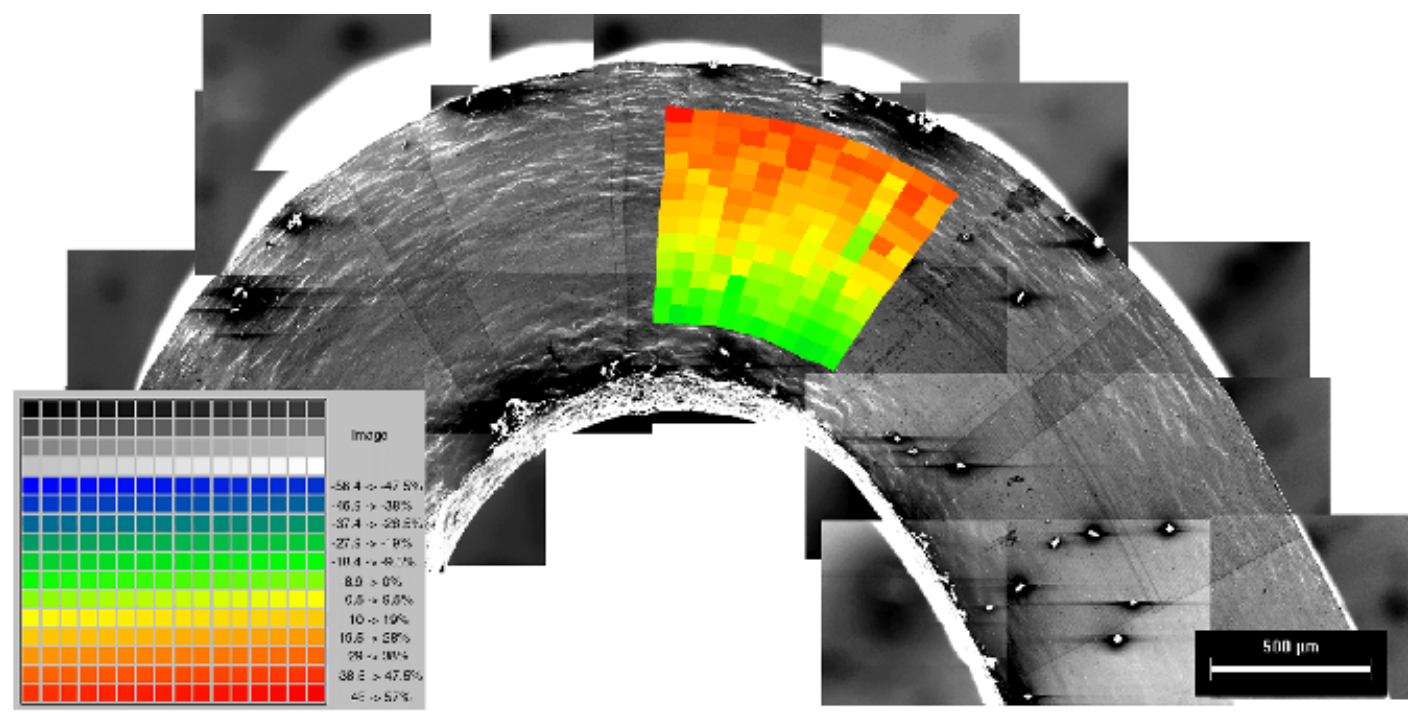

FIG. 2. SE image montage of a sheet specimen in cross section after bending and strain analysis. Local strain distribution and the location of the neutral axis are apparent.

FIG. 3. Comparison of empirical data with maximum surface strain values predicted by a Finite Element Model developed at Alcan.

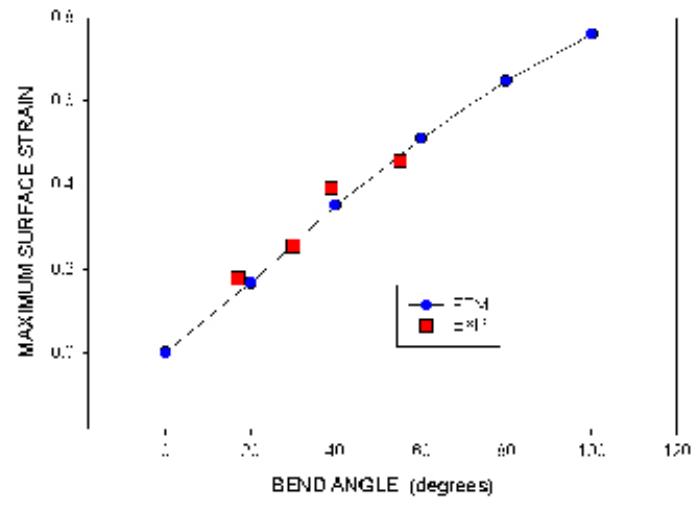

